\title{
Spatiotemporal interactions of predators and prey of a neotropical mammal community in southern Mexico
}

\author{
Miguel Briones-Salas ${ }^{1}$, Elena Galindo-Aguilar ${ }^{1}$, Beatriz Luna-Olivera ${ }^{2}$, Marcelino \\ Ramírez-Ibáñez ${ }^{3}$, and Mario C. Lavariega ${ }^{4}$ \\ ${ }^{1}$ Instituto Politécnico Nacional Centro Interdisciplinario de Investigación para el Desarrollo \\ Integral \\ ${ }^{2}$ Centro de Altos Estudios de la Mixteca, CALMIX, Oaxaca \\ ${ }^{3}$ CONACyT-UPN unidad 201 \\ ${ }^{4}$ Instituto Politecnico Nacional Centro Interdisciplinario de Investigacion para el Desarrollo \\ Integral
}

November 20, 2020

\begin{abstract}
Predator-prey interactions are one of the central themes in ecology due to their importance as a key mechanism in structuring biotic communities. In the predator-prey systems, the no-trophic interactions, such as persecution and avoidance, have more impact on ecosystems than the trophic ones. We analyze the spatiotemporal relations between prey and predators of a community of medium and large-size mammals in a tropical region. We analyzed time data series of camera-trap data. The observed data of time lags among occurrences of pairs of species were compared with random data using the Bootstrap method. We generate a network of co-occurrences to describe the significant spatiotemporal patterns between predators and prey and used the kernel density estimator to analyze the overlap of daily activity patterns themy. We found 26 predator-prey interactions $(\mathrm{p}<0.05)$, which involved 14 species. The results suggest that prey perceives the risk of predation and display avoidance behavior both spatially and temporally which is consistent with fear theory. Predators may be deploying opportunistic and / or intermittent foraging strategies to reduce prey mobility caused by previous predation encounters. This study provides a new approach to understand the interactions between predators and prey through camera-trapping or similar data of spatiotemporal co-occurrences.
\end{abstract}

\section{Hosted file}

Spatiotemporal interactions.pdf available at https://authorea.com/users/377482/articles/ 494142-spatiotemporal-interactions-of-predators-and-prey-of-a-neotropical-mammalcommunity-in-southern-mexico 

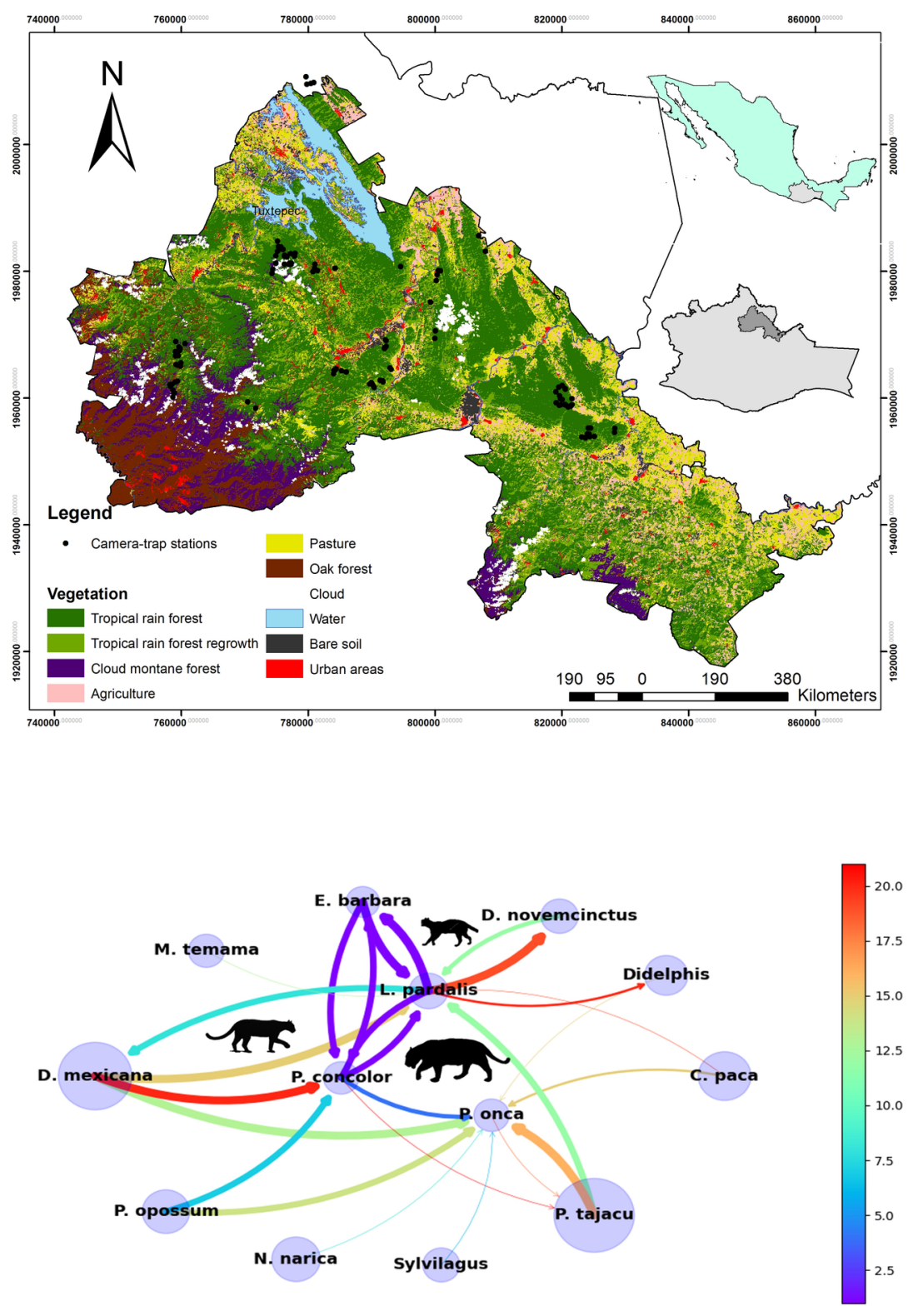